\title{
Review
}

\section{Antiangiogenic Steroids in Human Cancer Therapy}

\author{
Richard J. Pietras and Olga K. Weinberg
}

\author{
David Geffen School of Medicine at UCLA, Department of Medicine, Division of Hematology-Oncology, \\ Los Angeles, CA, USA
}

\begin{abstract}
Despite advances in the early detection of tumors and in the use of chemotherapy, radiotherapy and surgery for disease management, the worldwide mortality from human cancer remains unacceptably high. The treatment of cancer may benefit from the introduction of novel therapies derived from natural products. Natural products have served to provide a basis for many of the pharmaceutical agents in current use in cancer therapy. Emerging research indicates that progressive growth and spread of many solid tumors depends, in part, on the formation of an adequate blood supply, and this process of tumorassociated angiogenesis is reported to have prognostic significance in several human cancers. This review focuses on the potential application in antitumor therapy of naturally-occurring steroids that target tumor-associated angiogenesis. Squalamine, a 7,24 dihydroxylated 24-sulfated cholestane steroid conjugated to a spermidine at position $\mathrm{C}-3$, is known to have strong antiangiogenic activity in vitro, and it significantly disrupts tumor proliferation and progression in laboratory studies. Work on the interactions of squalamine with vascular endothelial cells indicate that it binds with cell membranes, inhibits the membrane $\mathrm{Na}^{+} / \mathrm{H}^{+}$exchanger and may further function as a calmodulin chaperone. These primary actions appear to promote inhibition of several vital steps in angiogenesis, such as blockade of mitogeninduced actin polymerization, cell-cell adhesion and cell migration, leading to suppression of endothelial cell proliferation. Preclinical studies with squalamine have shown additive benefits in tumor growth delay when squalamine is combined with cisplatin, paclitaxel, cyclophosphamide, genistein or radiation therapy. This compound has also been assessed in early phase clinical trials in cancer; squalamine was found to exhibit little systemic toxicity and was generally well tolerated by treated patients with various solid tumor malignancies, including ovarian, non-small cell lung and breast cancers. Clinical trials with squalamine alone or combined with standard chemotherapies or other biologic therapies, including antiangiogenic agents, should be considered for selected cancer patients, and further study of the mechanism of action and bioactivity of squalamine is warranted.
\end{abstract}

Keywords: malignancy - squalamine - tumor-associated angiogenesis - vascular endothelial growth factor (VEGF)

\section{Introduction}

Mortality from cancer, the second leading cause of death worldwide, remains unacceptably high. From 1975 to 2000, the total number of cancer cases worldwide rose from 5.9 million to 10 million, an increase of $69 \%$ (1). Although advances in the early detection of tumors and in the use of chemotherapy

For reprints and all correspondence: Richard J. Pietras, PhD, MD, UCLA School of Medicine, Department of Medicine-Hematology/Oncology, 11-934 Factor Building, Los Angeles, CA 90095-1678, USA. Tel: +1 310825 9769; Fax: +1 310825 6192; E-mail: rpietras@ucla.edu and surgery for disease management have helped to enhance the overall survival of afflicted patients, major improvements in treatments for most human cancers are urgently needed. The control and therapy of cancer may benefit from introduction of new treatments derived from natural products (2). Between 1974 and 1994, it is estimated that about 67\% of pharmaceutical products approved for human cancer therapy were derived from natural sources. The discovery of efficacious compounds for cancer management will benefit from new understanding of the molecular and cellular pathways that regulate tumor proliferation and progression.

(C) The Author (2005). Published by Oxford University Press. All rights reserved.

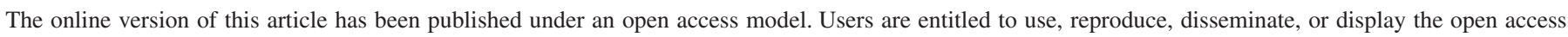

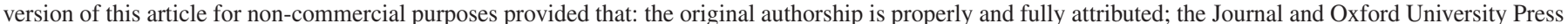

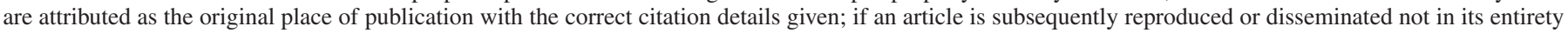
but only in part or as a derivative work this must be clearly indicated. For commercial re-use, please contact journals.permissions@ oupjournals.org 
The progressive growth and spread of many solid tumors depends, in part, on the formation of an adequate blood supply, and tumor angiogenesis has been reported to have prognostic significance in several human cancers (3). Therapy directed toward the vasculature of solid tumors is now being pursued as an important new direction in cancer treatment because avascular tumors exhibit only limited growth and tumor aggressiveness, and metastatic potential commonly correlates with tumor vascularity (4-7). Vascular endothelial growth factor (VEGF) is produced by most solid tumors and elicits a mitogenic effect on tumor-associated endothelial cells $(8,9)$. VEGF binding to receptor tyrosine kinases triggers activation of downstream signaling enzymes, including MAP kinase, which, in turn, regulate gene expression and specific endothelial cell responses including proliferation, migration and apoptosis (10-12). Several studies have suggested that VEGF plays an important role in the progression of many cancers $(3,13,14)$. Growth factor pathways, such as those dependent on EGF and HER-2 receptors, appear to up-regulate VEGF production in solid tumors $(15,16)$. Since EGF and HER family receptors are activated $(17,18)$ and/or overexpressed in significant numbers of human cancers (18-21), these growth factor receptor pathways may play a role in promoting further growth of human malignancy by increasing VEGF-dependent tumor angiogenesis.

\section{Antiangiogenic Steroids in Cancer Therapy}

Many small molecules, proteins and antibodies directed to growth factors have been identified as having antiangiogenic activity. Several steroids were among the first molecules recognized as having antiangiogenic activity. These compounds included progestin, medroxyprogesterone acetate and glucocorticoids such as dexamethasone and cortisone $(6,7)$. Such angiostatic steroids appear to have multiple and diverse effects on tumor-associated angiogenesis, including the inhibition of endothelial cell proliferation, the inhibition of collagenolysis and of plasminogen activator production, as well as direct antitumor activity amongst some malignancies. However, the activity of these compounds was characterized primarily by their ability to prevent new blood vessel growth in the chick embryo allantoic membrane assay system for angiogenesis. The most potent of these antiangiogenic steroids, $11 \alpha$-hydrocortisone and tetrahydrocortisol, lacked mineralocorticoid or glucocorticoid activity and produced capillary regression in the chick embryo allantoic membrane assay (22).

Later, using tumor models in animals, several antiangiogenic steroids exhibited measurable activity that was often enhanced when the steroid was given in combination with heparin or a heparin-like molecule (22-24). Of these antiangiogenic steroids, only medroxyprogesterone was assessed in clinical trials, primarily in patients with previously treated breast cancer (25). Study endpoints of partial or complete tumor responses showed only minimal clinical activity and no extension of survival.

An endogenous metabolite of estrogen, 2-methoxyestradiol, has been found to inhibit proliferation, migration and invasion of endothelial cells in vitro as well as having antiangiogenic effects in certain in vivo tumor models (26). The mechanism of action of this compound is not yet fully elucidated, but its action does not seem to be mediated by classical steroid hormone receptors. Recent evidence has shown that 2-methoxyestradiol inhibits HIF- $1 \alpha$, a key angiogenic transcription factor, and is, thus, able to elicit a broad spectrum of cellular effects. This activity appeared to correlate with microtubule-depolymerizing properties of 2-methoxyestradiol. This steroidal compound is also reported to initiate apoptosis in both vascular endothelial and solid tumor cells. In preclinical models, 2-methoxyestradiol reduced tumor size and tumor-associated vascularization. In early clinical trials, this agent appears to be well tolerated by patients with cancer, and use of 2-methoxyestradiol is currently being evaluated in several different types of malignancy (26).

Squalamine, a natural steroidal compound initially found in several tissues of the dogfish shark, Squalus acanthias, causes changes in vascular endothelial cell shape and has been reported to possess significant antiangiogenic activity in models of lung, breast, brain and ovarian cancer $(22,27,28)$. In the shark, squalamine is found primarily in sites of bile synthesis such as liver and gallbladder, but the aminosterol compound also occurs in smaller amounts in the spleen, testes, stomach, gills and intestine (29). In the laboratory, squalamine was originally found to have bactericidal activity against gram-negative and gram-positive bacteria, as well as some fungicidal qualities (29). More importantly, squalamine at relatively low doses was later shown to selectively inhibit the formation of new blood vessels. However, unlike previously described steroids, squalamine has significant structural differences and does not interact with glucocorticoid or minealocorticoid receptors (30). It is a 7,24 dihydroxylated 24-sulfated cholestane steroid conjugated to a spermidine at position C-3 (see Fig. 1). Squalamine has been demonstrated to be an angiostatic steroid by virtue of its inhibition of growth of vascular endothelial cells in culture, activity in the chick embryo chorioallantoic membrane assay and a rabbit corneal micropocket assay, as well as growth inhibition of gliomas and lung cancers in vivo $(22,28,31)$. Squalamine is somewhat unique among most current anti-angiogenic agents in development because it inhibits endothelial cell proliferation and migration induced by a wide variety of growth factors, including Basic Fibroblast Growth Factor (bFGF) and VEGF (27,31; Fig. 2). This broad antiangiogenic activity of squalamine may result from its inhibition of surface sodium-proton exchangers (thus altering intracellular $\mathrm{pH}$ and thereby impeding intracellular signaling by several growth factors) and other downstream signaling pathways in endothelial cells $(27,29)$. There are different theories about the mechanism of action of squalamine that remain to be investigated.

\section{Mechanism of Action of Squalamine}

One mechanism of action of squalamine has been proposed by Akhter et al. (32) to involve inhibition of the mammalian brush-border $\mathrm{Na}^{+} / \mathrm{H}^{+}$exchanger isoform NHE3. The $\mathrm{Na}^{+} / \mathrm{H}^{+}$ exchanger is a transport protein that is known to regulate 


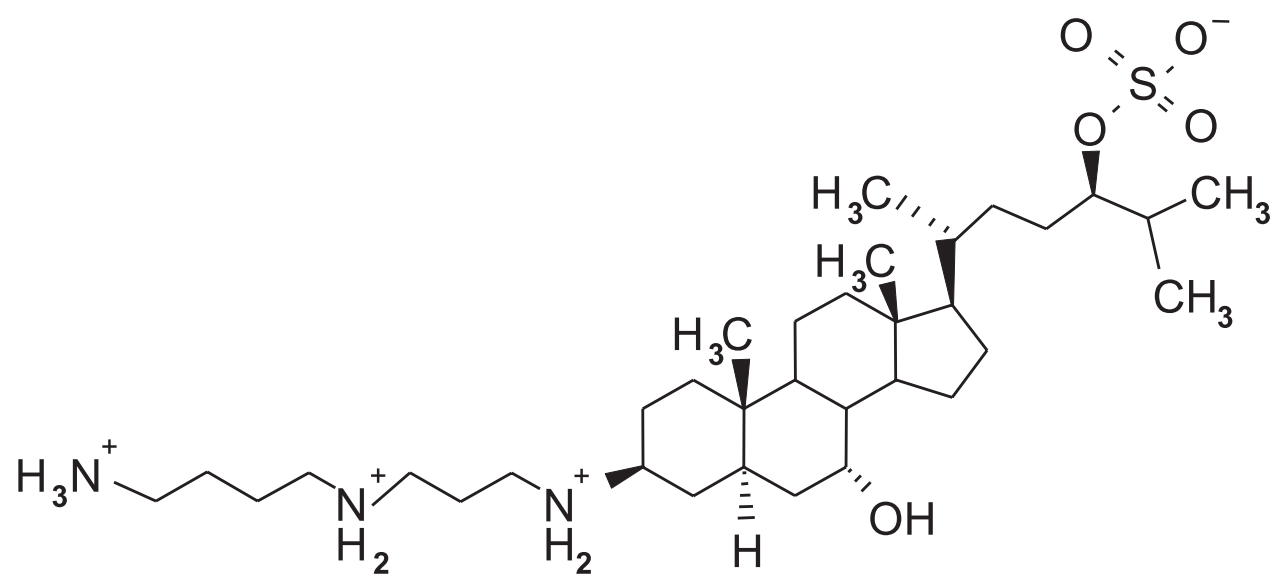

Figure 1. Squalamine, a 7,24 dihydroxylated 24-sulfated cholestane steroid conjugated to a spermidine at position C-3. This steroidal compound was initially found in tissues of dogfish shark, Squalus acanthias, primarily in sites of bile synthesis such as liver and gallbladder. However, squalamine also occurs in spleen, testes, stomach, gills and intestine. See text for additional details.

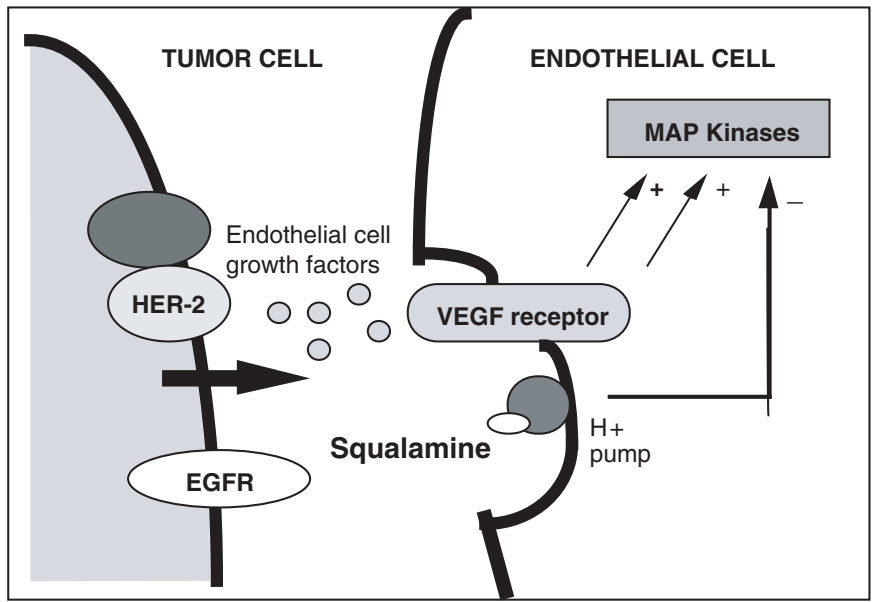

Figure 2. Models of tumor-associated angiogenesis in human solid tumors. Cancer cells with growth factor receptors (e.g. EGFR, HER-2) secrete endothelial cell growth factors that stimulate vascular endothelial cell growth factor (VEGF) receptors (4). Both peptide and steroid regulation of blood vessel growth may involve MAPK pathways that regulate the cytoskeleton and cell proliferation (53). Squalamine may interact with cell surface proton pumps, calmodulin or other signaling pathways, possibly localized in caveolaerelated lipid rafts $(27,29,32)$, to block activation of MAP kinases in blood endothelial cells. See text for details.

changes in cell volume or cell shape. Using transfected PS120 fibroblasts, the investigators found that inhibitory effects of squalamine on $\mathrm{Na}^{+} / \mathrm{H}^{+}$exchanger activity were both time and concentration dependent and reversible. The effect of the steroidal compound was measured as changes in intracellular $\mathrm{pH}$, specifically $\mathrm{Na}^{+}$-dependent alkalinization of the cell interior was inhibited by exposure to squalamine. Inhibition of $\mathrm{Na}^{+} / \mathrm{H}^{+}$exchange, as opposed to another mechanism of $\mathrm{pH}$ regulation, was confirmed by independent monitoring of ${ }^{22} \mathrm{Na}^{+}$ uptake by cells. This effect of squalamine was found to be specific for NHE3, as it did not affect NHE1, NHE2, $\mathrm{Na}^{+}-$ dependent D-glucose uptake or $\mathrm{Na}^{+}$-independent D-glucose uptake. To rule out that inhibitory effects of squalamine were not due to cytotoxicity, Lactate Dehydrogenase (LDH) release was also measured and found to be $<5 \%$ of total cell LDH. Using a truncated mutant of NHE3, this study also showed that inhibition of NHE3 required the C-terminal 76 amino acids of NHE3. This region of NHE3 is a critical part of the enzyme regulatory domain, with external control by calmodulin, calmodulin-kinase II, tyrosine kinases and squalamine. The inhibitory effect of calmodulin on NHE3 appears to be mediated through these $76 \mathrm{C}$-terminal amino acids as well. However, it remains undetermined if squalamine inhibition of NHE3 requires interaction with other regulatory molecules. Nonetheless, squalamine regulation of intracellular $\mathrm{pH}$ could have a major impact on the activity of other signaling pathways in target cells such as vascular endothelial cells.

Squalamine has also been proposed to be a calmodulin chaperon by Chen et al. (33). In efforts to isolate and partially purify squalamine-binding components from human placenta, Chen et al. determined the direct association of squalamine with calmodulin. Indeed, specific interactions between calmodulin and squalamine occurred for both forms of calmodulin, calcium-free and calcium-bound. Further, exposure of human vascular endothelial cells to fluorescent-tagged squalamine revealed a rapid relocalization of calmodulin and squalamine from the cell periphery to the perinuclear endosomal compartment. After squalamine treatment, F-actin stress fibers were disrupted and a change in cell shape was noted. Based on these observations, the authors proposed that the mechanism of action of squalamine involved its specific entry into endothelial cells, leading to the intracellular redistribution of calmodulin without inhibition of calmodulin function.

The biologic actions of squalamine were assessed further by Sills et al. (28). In this work, squalamine was found to inhibit rat brain endothelial cell proliferation and migration induced by mitogens such as VEGF, bFGF, Platelet Derived Growth Factor (PDGF), and scatter factor/hepatocyte growth factor. In the absence of these mitogens, squalamine was found to have no direct effect on survival or proliferation of endothelial cells. 
In addition, squalamine was also found to inhibit proton secretion by mitogen-stimulated endothelial cells, a finding consistent with results reported by Akhter et al. (32). An interesting finding of this study involved the direct application of squalamine to 4-day-old chick embryo vasculature. After only 20 min, squalamine elicited constriction of the smallest capillaries throughout the yolk sac, with entrapment of red cells. In addition, the usual continuous blood flow through large vessels was changed to a pulsatile expansion in order to move through occluded vessels. This acute remodeling process resulted in narrowed vascular segments and blocked erythrocyte movement and was confirmed by histological examination of treated and untreated yolk sacs. Since these new vessels are composed solely of endothelial cells, the luminal narrowing was concluded to be due to squalamine-induced changes in the shape or volume of endothelial cells. This study also established that systemic treatment with squalamine promoted an inhibition of the growth of rat flank 9L glioma tumors. Immunohistochemical analyses of these tumors after treatment with squalamine revealed significant reductions in tumor-associated bloodvessel density.

In order to further understand the molecular and cellular mechanisms by which squalamine inhibits tumor growth, Williams et al. (34) evaluated the effects of squalamine on the organization of the endothelial cell actin cytoskeleton and transmembrane cell-cell adhesion elements. Human vascular endothelial cells were grown and stimulated with VEGF and then treated with squalamine. The results showed that squalamine disrupted polymerization of F-actin fibers in these cells. In addition, after squalamine treatment, it was found that significantly less VE-cadherin was detectable at the endothelial cell-cell junction, with apparent increments in peri-nuclear VE-cadherin. This suggested that squalamine induced an internalization process that involves moving VE-cadherin from the membrane into the intracellular compartment. VE-cadherin is a member of the cadherin family that is involved in cell-cell adherence junction maintenance and is also associated with actin filament attachment sites on the cell surface. These findings led the authors to conclude that squalamine perturbs both actin polymerization and cell-cell adhesion in endothelial cells. Thus, squalamine interferes with the ability of endothelial cells to move, grow and communicate, thus inhibiting the formation of new vessels. This process may be very important in preventing the nourishment and progression of tumors.

Li et al. (15) conducted studies of human ovarian tumorassociated angiogenesis. Ovarian cells were found to secrete significant levels of VEGF, a direct activator of angiogenesis (see Fig. 2), but squalamine did not reduce VEGF secretion by tumor cells, and it evoked no direct growth inhibition of ovarian cells in vitro. However, squalamine at doses as low as $160 \mathrm{nM}$ did halt the proliferation of human vascular endothelial cells and markedly reduced VEGF-induced capillary tubelike formations by vascular endothelial cells growing in Matrigel culture (35). These biologic effects correlated well with squalamine-induced blockade of the rapid VEGF-stimulated phosphorylation of p44/p42 MAP kinase in endothelial cells, an early cell response to activate proliferation. VEGF is considered to exert its biologic effects by binding with receptor tyrosine kinases, notably Flt-1 and Flk-1/KDR, present at the surface of endothelial cells $(36,37)$. Post-receptor signal transduction regulates the effects of VEGF (see Fig. 2), and the proliferative action of VEGF in endothelial cells has been associated, in part, with VEGF-induced tyrosine phosphorylation and stimulation of MAP kinase, ERK-1 (p44) and ERK-2 (p42) (10,37). Of note, squalamine also reduced VEGFinduced phosphorylation of focal adhesion kinase (FAK) and stress-activated protein kinase-2/p38 SAPK2, blocking, in turn, assembly of F-actin stress fibers in vascular endothelial cells. These effects follow primary interaction of squalamine with caveolar domains at the surface membranes of endothelial cells, sites for the concentration of vital signaling complexes to regulate the angiogenic process (37). Squalamine interference with these downstream signaling pathways in vascular endothelial cells may be critical in disrupting the process of tumor-associated angiogenesis.

\section{Preclinical Studies with Squalamine}

Studies by Teicher et al. (22) noted that squalamine as a single agent has a modest effect on tumor growth delay on rat 13762 mammary carcinoma, with squalamine dosing at $40 \mathrm{mg} / \mathrm{kg}$. Combining squalamine with cyclophosphamide, cisplatin or paclitaxel increased the tumor growth delay by 1.9-2.5-fold as compared to any of these agents administered alone. A Lewis lung carcinoma was a second tumor model examined in this study. The lung tumor model allowed squalamine effects on primary and metastatic disease to be evaluated since untreated mice generally die from metastatic tumor spread by days 21-25. Squalamine alone had a modest effect on tumor growth delay, but, again, an additive effect was seen when squalamine was combined with cyclophosphamide, cisplatin, paclitaxel or 5-fluorouracil. Moreover, it was found that the number of lung metastases decreased when mice were treated with squalamine. Specifically, by day 20, the numbers of metastases were reduced to half of those present in controls. Since lung metastases are actively implanting and growing using new blood vessels, this effect of squalamine suggests that it has strong antiangiogenic potency.

Using non-small cell lung tumor models, Williams and colleagues (34) assessed the effect of squalamine alone and combined with either cisplatin or paclitaxel with carboplatin. Chemotherapy-resistant MV-522 tumor cells were implanted as xenografts in immunodeficient mice and allowed to reach a size of about $60 \mathrm{mg}$. Squalamine was administered alone at dose of $20 \mathrm{mg} / \mathrm{kg} /$ day or in combination with cisplatin (dose of $2 \mathrm{mg} / \mathrm{kg} /$ day) or as a part of triple therapy with paclitaxel (10 mg/kg/day) and carboplatin $(20 \mathrm{mg} / \mathrm{kg} /$ day $)$. The results indicated that squalamine either combined with cisplatin or included as a part of triple therapy with paclitaxel and carboplatin was more effective than administration alone in suppressing the growth of MV-522 human lung tumors. Final excised mice tumor weight after treatment with squalamine 
alone was $613.6 \pm 64.6 \mathrm{mg}$, while treatment with squalamine and cisplatin resulted in final tumor weights of $105.0 \pm$ $28.6 \mathrm{mg}$. When administered as a part of triple therapy with paclitaxel and carboplatin, the final excised mice tumor weight was $56.4 \pm 14.5 \mathrm{mg}$. The effect of adding squalamine to other treatments was found to persist throughout the experiment.

The antitumor effects of squalamine with and without cytotoxic agents were examined in other human lung cancer xenografts by Schiller and Bittner (38). The cell lines used included Calu-6 (adenocarcinoma), H460 (large cell carcinoma), NL20T-A (derived from an immortalized human bronchial epithelial cell line that underwent spontaneous transformation to a tumorigenic phenotype) and Lewis lung. Squalamine appeared to have no cytotoxic effect in vitro on lung cell lines, and addition of squalamine to cisplatin did not result in enhanced death of tumor cells. In vivo, squalamine alone was effective in reducing tumor formation only when administered before, simultaneously, or $24 \mathrm{~h}$ after inoculation of mice with tumor cells. When combined with platinum analogs, squalamine resulted in 1.5-2-fold growth delay of tumors in all four lung cell lines. No antitumor activity was seen when squalamine was combined with paclitaxel, venorelbine, gemcitabine or docetaxel. Multiple doses of cisplatin and squalamine increased tumor growth delay more than a single dose. Using H460 tumor xenografts, the effects of squalamine on tumor vascularization were determined. As a single agent, squalamine had no effect on the number of CD31-staining vessels in established tumors. However, when used in combination with cisplatin, the number of vessels was reduced by $25 \%$. This effect was observed early (within $2-4 \mathrm{~h}$ after treatment) and dissipated by $48 \mathrm{~h}$. These results suggest that squalamine and platinum analogs likely need to be administered together in close temporal proximity.

In studies of human non-small cell lung tumors grown as xenografts in nude mice, our laboratory has further assessed the effects of squalamine treatment alone and combined with either the phytoestrogen, genistein, or radiation therapy (see Fig. 3). The non-small cell lung tumor, NCI-H23, as many human lung tumors, is enriched with estrogen receptor- $\beta$ and this receptor signaling pathway may also promote angiogenesis (39). Treatment with squalamine alone elicits a modest inhibition of the growth of human non-small cell lung tumor cells NCI-H23 $(P<0.05)$. Moreover, combination of squalamine with genistein (Fig. 3A), a potential inhibitor of estrogen receptor- $\beta$ activity, appears to elicit additive antitumor effects that exceed the responses to single agent treatments $(P<$ 0.001 ). In addition, squalamine was combined with radiation therapy, a common treatment intervention for non-small cell lung cancer, and this combination was highly effective in suppressing tumor growth $(P<0.001)$ (Fig. 3B). This latter finding supports independent data suggesting that the combination of ionizing radiation with antiangiogenic agents can improve tumor eradication, possibly without increasing deleterious effects (40).

Squalamine's effect on breast cancer was studied by Williams et al. (41). MX-1 breast xenografts were grown in mice and were treated with Cytoxan (cyclophosphamide). After tumor regression, mice were randomized into control and squalaminetreated groups. Two different doses of squalamine were used (10 and $20 \mathrm{mg} / \mathrm{kg} /$ day) and both resulted in increased numbers
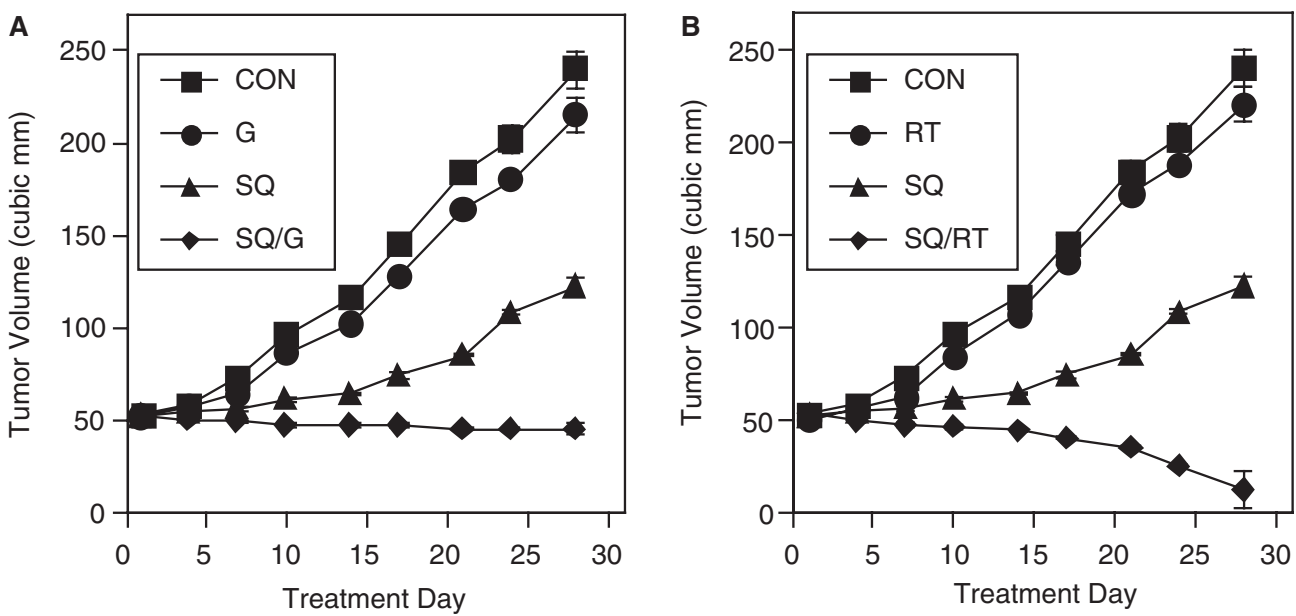

Figure 3. Squalamine inhibits growth of human non-small cell lung tumor cells and enhances the antitumor effects of radiation therapy and genistein. Lung cancers with estrogen receptor- $\alpha$ and estrogen receptor- $\beta$ expression (NCI-H23) were grown as subcutaneous xenografts to $50-100 \mathrm{~mm}^{3}$ in size in ovariectomized, nude mice primed with estrogen $(1.7 \mathrm{mg} / \mathrm{pellet})$. Mice were then treated as outlined here. (A) Genistein, a phytoestrogen, and squalamine inhibit growth of human lung tumor xenografts NCI-H23 in nude mice. Mice with established tumors were treated with control solution, genistein alone $(0.2 \mathrm{mg} / \mathrm{kg}$ s.c. on alternate days) (18), squalamine alone ( $2 \mathrm{mg} / \mathrm{kg}$ s.c.) on days $1-10$, or genistein with squalamine. By 28 days, tumors showed little tumor regression after treatment with genistein alone and modest regression after squalamine alone compared to control $(P<0.05)$. More profound tumor growth inhibition was elicited by treatment with squalamine plus genistein $(P<0.001)$. Mean tumor volumes of control $(\mathrm{CON})$, genistein $(\mathrm{G})$, squalamine $(\mathrm{SQ})$ and combined squalamine/genistein $(\mathrm{SQ} / \mathrm{G})$ treatment are shown. (B) Radiation therapy (RT) and squalamine inhibit growth of lung tumor xenografts NCI-H23 in nude mice. Mean tumor volumes of control (CON), RT (4 Gy on days 1, 4 and 7), squalamine (SQ; $2 \mathrm{mg} / \mathrm{kg}$ s.c. on days 1-10) and combined squalamine/RT (SQ/RT) treatment are shown. See Pietras et al. $(54,55)$ and text for details. 
of tumors which underwent complete regression. The authors conclude that squalamine influences the latent period for tumor expansion post effective chemotherapy.

Previous studies have suggested that VEGF plays an important role in progression of ovarian cancer (15). Ovarian cancer is the most deadly gynecologic malignancy (42-45). Although advances in chemotherapy and surgery have helped to improve the overall survival of afflicted patients, 5-year survival rates from ovarian cancer remained about $44 \%$ in the early part of this decade. By the time many patients are diagnosed with ovarian cancer, peritoneal dissemination of the tumor has often occurred. This growth and spread of ovarian cancers depends, in part, on formation of an adequate blood supply. Tumorassociated angiogenesis is essential for growth of most solid tumors, and neovascularization has also been shown to have prognostic significance in epithelial ovarian cancer (3). Several studies have shown that VEGF expression plays an important role in the progression of ovarian cancers $(3,13,14)$, and the further ability of VEGF to increase vascular permeability $(4,8,46)$ may contribute to formation of malignant ascites in patients with ovarian cancer (47). It is possible that treatment strategies directed to suppress the expression of VEGF and related angiogenic molecules may help to block tumor progression and reduce the morbidity associated with malignant ascites. Since squalamine has been shown to inhibit endothelial proliferation by mitogens such as VEGF, the effect of squalamine on ovarian cancer was examined by $\mathrm{Li}$ et al. (15). Using human ovarian OV2008 cells with HER-2 overexpression grown as xenografts in mice, the authors were able to show profound tumor growth inhibition by combined treatment with squalamine and cisplatin (see Fig. 4). This growth inhibition persisted for 18 days after

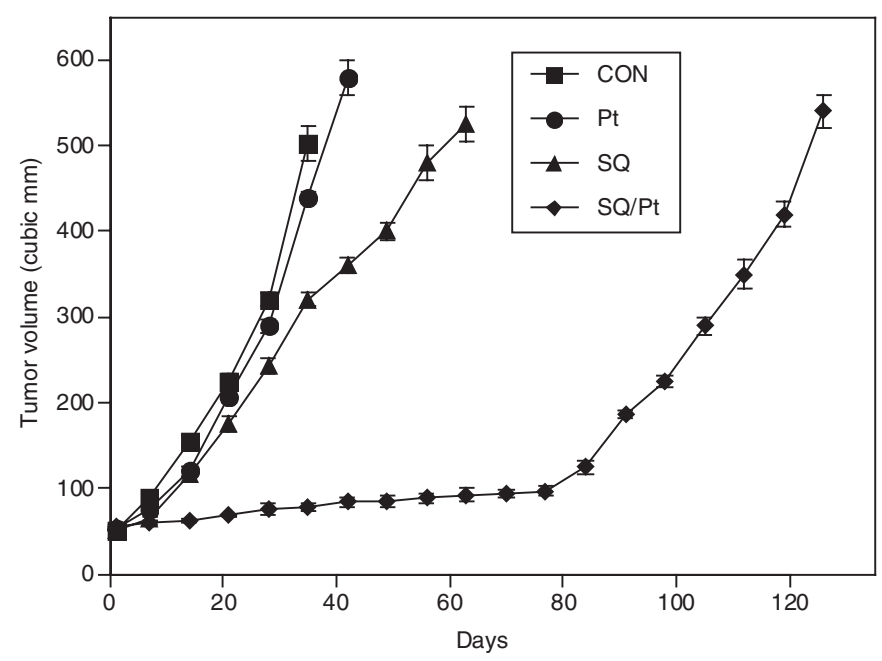

Figure 4. Squalamine promotes the time to tumor progression in a human ovarian tumor xenograft model in vivo. CAOV3 ovarian cancer cells were inoculated subcutaneously in nude mice as before (15). After 7 days, animals with tumors of comparable size were randomized to treatment with control solution $(\mathrm{CON})$, squalamine (SQ; $2 \mathrm{mg} / \mathrm{kg}$ on days $1-10)$, cisplatin $(\mathrm{Pt}$; $4 \mathrm{mg} / \mathrm{kg}$ on day 1 ), or cisplatin administered in combination with squalamine $(\mathrm{SQ} / \mathrm{Pt})$. Tumor volumes $\left(\mathrm{mm}^{3}\right)$ are expressed as mean $\pm \mathrm{SEM}$ for measurements to assess tumor growth delay due to the several treatments. Data derived from Li et al. (15), with permission. stopping squalamine treatment. This study showed that the length of tumor growth delay was greatest with the combined treatment of squalamine and cisplatin as compared with the effects of single-agent therapy.

The molecular effects of squalamine and cisplatin were also assessed by evaluating levels of tumor cell apoptosis. Administration of squalamine in combination with cisplatin led to enhanced levels of apoptosis in several ovarian tumor cells assessed in vivo. The extent of tumor cell death was higher with the combination treatment than with either squalamine or cisplatin administered alone. The findings suggest that squalamine enhances the cytotoxicity of cisplatin chemotherapy by facilitating tumor cell apoptosis. Using immunohistochemical methods to assess tumor-associated blood vessel density, the investigators were able to show that squalamine caused a reduction of tumor-associated angiogenesis in different types of ovarian tumors.

\section{Clinical Studies with Squalamine}

On the basis of strong evidence of antiangiogenic and antitumor properties of squalamine, it was selected for clinical development as a therapeutic agent for treatment of human malignancies. The squalamine in use in these and more recent clinical trials is synthesized in a pharmaceutical facility (48). A phase I study was conducted by Bhargava et al. (49) to determine the maximum tolerated dose (MTD), dose-limiting toxicities (DLTs) and the pharmacokinetic profile of squalamine. The investigators recruited 19 patients with an Eastern Cooperative Oncology Group (ECOG) performance status of $\leq 2$ with advanced non-leukemic cancers. Squalamine was administered as a continuous intravenous infusion over $120 \mathrm{~h}$, with repeat dosing every 14 days. The starting dose was chosen as $6 \mathrm{mg} / \mathrm{m}^{2} /$ day, increasing at a rate of $100 \%$ up to a dose of $384 \mathrm{mg} / \mathrm{m}^{2} /$ day, after which it was escalated in $40 \%$ increments until DLT was encountered. The highest dose administered was $538 \mathrm{mg} / \mathrm{m}^{2} /$ day. The best-tolerated dose of squalamine was found to be $192 \mathrm{mg} / \mathrm{m}^{2} /$ day, although a dose of $384 \mathrm{mg} / \mathrm{m}^{2} /$ day also appeared to be well-tolerated in patients without prior exposure to squalamine. Three or more patients were treated at each dose level. In the absence of toxicity during the first treatment cycle, patients were allowed to have their doses escalated. This reduced the time to reach the MTD, with less patients required to start at lower doses. The DLT noted with squalamine administration was hepatotoxicity, measured as abnormalities in liver function tests and mostly noted to occur at the highest dose of $538 \mathrm{mg} / \mathrm{m}^{2} /$ day. However, the hepatotoxicity was found to be transient and reversible. Mild to moderate fatigue was also noted in all patients at all dose levels, and this resolved after treatment. Plasma pharmacokinetics from this study showed that squalamine has a short initial elimination half-life and a high plasma clearance. The area under the concentration-time curve $\left(\mathrm{AUC}_{0-\mathrm{t}}\right)$ and the maximum concentration $\left(\mathrm{C}_{\max }\right)$ of squalamine was found to be dose and dose-rate proportional over $12-384 \mathrm{mg} / \mathrm{m}^{2} /$ day, with evidence of nonlinearity at $538 \mathrm{mg} / \mathrm{m}^{2} /$ day. Clearance of squalamine was also 
unchanged at all doses. Transient tumor regression was observed in some patients treated with squalamine, but all patients had overall progression of their disease. The investigators emphasize that all patients in this trial had advanced malignant disease, while antiangiogenic treatments are hypothesized to work best in individuals with minimal disease burden. In addition, maximal squalamine effects are most likely obtained when combined with chemotherapy drugs as demonstrated in preclinical studies. In general, the treatment with squalamine was well-tolerated by patients.

Another phase I and pharmacokinetic study of squalamine was reported by Hao et al. (50). They recruited 33 patients with advanced solid tumor malignancies. Squalamine was administered as a 5-day continuous intravenous infusion that was repeated every 3 weeks. The starting dose was $6 \mathrm{mg} / \mathrm{m}^{2} /$ day, and dose levels were escalated in $40 \%$ increments. The maximal dose administered was $700 \mathrm{mg} / \mathrm{m}^{2} /$ day. The main DLT in this trial was also found to be elevations in hepatic transaminases and hyperbilirubinemia. At a dose of $700 \mathrm{mg} / \mathrm{m}^{2} /$ day, two out of three patients developed grade 4 hyperbilirubinemia and grade 3 or 4 elevations in hepatic transaminases. Based on these results, the authors concluded that the recommended dose of squalamine should not exceed $500 \mathrm{mg} / \mathrm{m}^{2} /$ day as a continuous intravenous infusion over 5 days every 3 weeks. This study evaluated a larger number of patients in the $357-700 \mathrm{mg} / \mathrm{m}^{2}$ dose range, as compared to the previous phase I study by allowing a different definition for DLT (e.g. grade 3 or 4 transaminase elevation lasting $>7$ days). This also led to somewhat different findings on squalamine kinetics. Squalamine was shown to exhibit dose-proportional kinetics in this study, whereas Bhargava et al. (49) concluded that $\mathrm{AUC}_{0-\mathrm{t}}$ and $\mathrm{C}_{\max }$ were nonlinear at the highest dose. With the 12-15-week treatments, stable disease was noted in some of the test patients, but no major tumor regressions were observed.

Since squalamine has shown evidence of additive or synergistic antitumor activity with certain cytotoxic chemotheurapeutic agents, a phase I/IIA study was designed by Herbst et al. (51) to examine the antitumor efficacy of squalamine in combination with carboplatin and paclitaxel in patients with a diagnosis of non-small cell lung cancer. The primary goal of this study was to examine safety, clinical response and pharmacokinetics of squalamine when administered as a 5-day continuous infusion in combination with standard chemotherapy in patients with stage IIIB or stage IV non-small cell lung cancers. With 45 patient volunteers, squalamine was administered as 5-day continuous intravenous infusion ranging from 100 to $400 \mathrm{mg} / \mathrm{m}^{2} /$ day repeated every 21 days for one cycle, at which point an evaluation of DLT was made. In the absence of DLT, dose escalation was permitted by $100 \mathrm{mg} / \mathrm{m}^{2} /$ day. Paclitaxel was administered at a dose of $225 \mathrm{mg} / \mathrm{m}^{2}$ as a 3-h i.v. infusion, followed by carboplatin and followed by squalamine. After one patient at the $400 \mathrm{mg} / \mathrm{m}^{2}$ dose experienced significant DLT, it was determined that $300 \mathrm{mg} / \mathrm{m}^{2} /$ day was the MTD for squalamine to be used in the phase II study. This dose was administered for up to six 21-day cycles. Importantly, significant toxicities in this study were limited to mild myelosuppresion. This study showed that squalamine exhibited linear pharmacokinetics, and plasma clearance of squalamine did not change at the doses used. Moreover, the pharmacokinetic profile of paclitaxel was not changed by combining the chemotherapy drug with squalamine. Similarly, carboplatin drug clearance was not changed by squalamine. With regard to antitumor efficacy endpoints, a partial clinical response was obtained in $28 \%$ of evaluable patients. Stable disease was shown in $19 \%$ of patients, while disease progression occurred in $53 \%$ of patients. Thus, significant clinical benefit was observed for $47 \%$ of evaluable patients with advanced stage non-small cell lung cancer. In the group of patients who were treated at the MTD of $300 \mathrm{mg} / \mathrm{m}^{2} /$ day, median survival was 8.5 months, and 1-year survival was $33 \%$.

To assess the antitumor efficacy of squalamine in human ovarian cancer, a phase II clinical study was performed by Davidson et al. (52). On recruiting 33 patients with stage III or stage IV ovarian cancer who were resistant or refractory to platinum-based chemotherapy, squalamine was administered with carboplatin as a 5-day continuous infusion at a dose of $200 \mathrm{mg} / \mathrm{m}^{2} /$ day. Average treatment time was 81 days. Response data have been reported for 22 patients, and, among this group, eight patients had an objective clinical response. Major toxicity reported in this study among three patients was grade 4 thrombocytopenia, anemia, leukopenia, myalgia or asthenia, symptoms often associated with carboplatin chemotherapy. Additional patients were enrolled in the study, but the final data remain to be presented (52). Nonetheless, these results suggest that squalamine may be a significant addition to current treatment options for patients with advanced refractory ovarian cancers. Based on currently-available information on the potential antitumor efficacy of squalamine in ovarian cancer, the antiangiogenic steroid has been designated an 'orphan drug' candidate for future therapeutic development in ovarian malignancy, a group for whom new treatment alternatives are urgently needed.

\section{Conclusions}

Natural products have served to provide a basis for many of the pharmaceutical agents in current use in cancer therapy and prevention. Squalamine, a natural steroidal compound, causes changes in vascular endothelial cell shape, and has been reported to possess significant antiangiogenic activity in models of lung, breast, brain and ovarian cancer. The mechanism of action has also been proposed to involve inhibition of endothelial cell proliferation and migration induced by mitogens in addition to being a calmodulin chaperone $(28,33)$. Preclinical studies with squalamine have shown that it is more effective in tumor growth delay when combined with radiation therapy, genistein, cisplatin, paclitaxel and cyclophosphamide. In addition, studies using Lewis lung carcinoma found that the number of metastasis were reduced by half after treatment, which confirms the antiangiogenic potency of squalamine. Squalamine exhibited little systemic toxicity in Phase I-II clinical trials and is well tolerated by treated cancer patients 
$(49,50,52)$. Pending positive results in future randomized, controlled clinical trials of antitumor efficacy, squalamine has the potential to become a useful addition to the clinical options available for patients with solid tumor malignancies, such as ovarian, non-small cell lung and breast cancers. Clinical trials with squalamine alone or combined with standard chemotherapies or other biologic therapies, including antiangiogenic agents, should be considered for selected cancer patients. The US Food and Drug Administration has designated squalamine as an 'orphan drug' for development as a new antitumor agent to treat ovarian cancer, and further study of its mechanism of action and bioactivity is warranted. The development of squalamine as an antitumor pharmaceutical provides yet another example of how a natural product can help to elucidate novel approaches to the treatment of disease.

\section{Acknowledgements}

This work was supported by grants from the US Department of Defense Ovarian Cancer Research Program, the US Department of Defense Breast Cancer Research Program, the NIH Lung Cancer SPORE Program at UCLA, the Ovarian Cancer Research Fund, the Stiles Program in Integrative Oncology (in vitro studies) and a Howard Hughes Fellowship (to O.K.W.). We thank Dr Kenneth Holroyd and Dr Jon Williams of Genaera Pharmaceuticals for providing synthetic squalamine for use in this work. Dr Manuel Gorrin-Rivas, Dr Diana Marquez, Ms Hsiao-Wang Chen, Mr Eugene Tsai, Dr Mike McLane and Dr Hsiao-Ling Hung provided useful advice and assistance.

\section{References}

1. Parkin DM. Global cancer statistics in the year. Lancet Oncol 2001;2:596.

2. Reddy L, Odhav B, Bhoola KD. Natural products for cancer prevention: a global perspective. Pharmacol Therapeut 2003;99:1-13.

3. Alvarez AA, Krigman HR, Whitaker RS, Dodge RK, Rodriguez GC. The prognostic significance of angiogenesis in epithelial ovarian carcinoma. Clin Cancer Res 1999;5:587-91.

4. Ferrara N, Winer, J, Burton T, et al. Expression of vascular endothelial growth factor does not promote transformation but confers a growth advantage in vivo to Chinese Hanster Ovary cells. J Clin Invest 1993;91:160-70.

5. Folkman J, Haudenschild C. Angiogenesis in vitro. Nature 1980;288:551-6.

6. Folkman J, Ingber D. Inhibition of angiogenesis. Sem Cancer Biol 1992; 3:89-96.

7. Folkman J, Shing Y. Angiogenesis. J Biol Chem 1992;267:10931-4.

8. Keck P, Hauser S, Krivi G, et al. Vascular permeability factor, an endothelial cell mitogen related to PDGF. Science 1989;240:1309-12.

9. Leung D, Cachianes G, Kuang WJ, Goeddel D, Ferrara N. Vascular endothelial growth factor is a secreted angiogenic mitogen. Science 1989;240:1306-9.

10. Rousseau S, Houle F, Landry J, Huot J. P38 MAP kinase activation by vascular endothelial growth factor mediates actin reorganization and cell migration in human endothelial cells. Oncogene 1997;15:2169-77.

11. Rousseau S, Houle F, Korarides H, et al. Vascular endothelial growth factor (VEGF)-driven actin-based motility is mediated by VEGFR2 and requires concerted activation of stress-activated protein kinase 2 (SAPK2/p38) and geldanamycin-sensitive phosphorylation of focal adhesion kinase. $J$ Biol Chem 2000;275:10661-72.

12. Soker S, Fidder H, Neufeld G, Klagsbrun M. Characterization of novel vascular endothelial growth factor (VEGF) receptors on tumor cells that bind VEGF165 via its exon 7-encoded domain. J Biol Chem 1996;271:5761-7.

13. Paley PJ, Staskus KA, Gebhard K, et al. Vascular endothelial growth factor expression in early stage ovarian carcinoma. Cancer 1997; 80:98-106.

14. Yamamoto S, Konishi I, Mandai M, et al. Expression of vascular endothelial growth factor (VEGF) in epithelial ovarian neoplasms: correlation with clinicopathology and patient survival, and analysis of serum VEGF levels. Br J Cancer 1997;76:1221-7.

15. Li D, Williams J, Pietras R. Squalamine and cisplatin block angiogenesis and growth of human ovarian cancer cells with or without HER-2 gene overexpression. Oncogene 2002;21:2805-14.

16. Petit AM, Rak J, Hung MC, et al. Neutralizing antibodies against epidermal growth factor and ErbB-2/neu receptor tyrosine kinases downregulate vascular endothelial growth factor production by tumor cells in vitro and in vivo: angiogenic implications for signal transduction therapy of solid tumors. Am J Pathol 1997;151:1523-30.

17. Gilmour L, Macleod K, McCaig A, et al. Neuregulin expression, function and signaling in human ovarian cancer cells. Clin Cancer Res $2002 ; 8: 3933-42$.

18. Hellstrom I, Goodman G, Pullman J, Yang Y, Hellstrom KE. Overexpression of HER-2 in ovarian carcinomas. Cancer Res 2001;61:2420-3.

19. Slamon DJ, Clark GM, Wong SJ, Levin WJ, Ulrich A, McGuire WJ. Human breast cancer: Correlation of relapse and survival with amplification of the HER-2/neu oncogene. Science 1987;235:177-82.

20. Slamon DJ, Godolphin W, Jones LA, et al. Studies of the HER-2/neu proto-oncogene in human breast and ovarian cancer. Science 1989;244:707-12.

21. Wong YF, Cheung TH, Lam SK, et al. Prevalence and significance of HER-2/neu amplification in epithelial ovarian cancer. Gynecol Obstet Invest 1995;40:209-12.

22. Teicher BA, Williams JI, Takeuchi H, Ara G, Herbst R, Buxton D. Potential of the aminosterol, squalamine in combination therapy in the rat 13762 mammary carcinoma and the murine Lewis Lung Carcinoma. Anticancer Res 1998;18:2567-74.

23. Folkman J, Langer R, Linhardt RJ, Haudenschild C, Taylor S. Angiogenesis inhibition and tumor regression caused by heparin or a heparin fragment in the presence of cortisone. Science 1983;221:719-25.

24. Sakamoto N, Tanaka NG, Tohgo A, Osada Y, Ogawa H. Inhibitory effects of heparin plus cortisone acetate on endothelial cell growth both in cultures and in tumor masses. J Natl Cancer Inst 1987;78:581-5.

25. Jakobsen A, Frederiksen PL, Moller KA, et al. Medroxyprogesterone acetate and prednisone in advanced breast cancer. A randomized trial. Eur J Cancer Clin Oncol 1986;22:1067-72.

26. Mooberry SL. Mechanism of action of 2-methoxyestradiol: new developments. Drug Resist Updates 2003;6:355-61.

27. Eckhardt, SG. Angiogenesis inhibitors as cancer therapy. Hospital Pract 1999;1:63-78.

28. Sills AK Jr, Williams JI, Tyler BM, et al. Squalamine inhibits angiogenesis and solid tumor growth in vivo and perturbs embryonic vasculature. Cancer Res 1998;58:2784-92.

29. Moore KS, Wehrli S, Roder H, et al. Squalamine: an aminosterol antibiotic from the shark. Proc Natl Acad Sci USA 1993;90:1354-8.

30. Bruss K, editor. American Cancer Society Guide to Complementary and Alternative Cancer Methods. Atlanta, GA: American Cancer Society, 2000.

31. Williams JI. Squalamine-A New Angiostatic Steroid. In Teicher BA, editor: Antiangiogenic Agents. Totowa, NJ: Humana Press, 1999.

32. Akhter S, Nath SK, Tse CM, William J, Zasloff M, Donowitz M. Squalamine, a novel cationic steroid, specifically inhibits the brushborder $\mathrm{Na}+/ \mathrm{H}+$ exchanger isoform NHE3. Am J Physiol 1999; 276:C136-44.

33. Chen Q, William JI, Anderson M, Kinney WK, Zasloff M. The angiostatic sterol squalamine is a calmodulin chaperone. Clin Cancer Res 1999;5 (Suppl):3768s.

34. Williams JI, Weitman S, Gonzalez CM, et al. Squalamine treatment of human tumors in $n u / n u$ mice enhances platinum-based chemotherapies. Clin Cancer Res 2001;7:724-33.

35. Pietras RJ, Gorrin-Rivas M, Chen H-W. Squalamine blocks tumorassociated angiogenesis and growth of ovarian cancer. Int J Obstet Gynecol 2003;83(Suppl 3):59-60.

36. Mustonen T, Alitalo K. Endothelial receptor tyrosine kinases involved in angiogenesis. J Cell Biol 1995;129:895-8.

37. Soker S, Fidder H, Neufeld G, Klagsbrun M. Characterization of novel vascular endothelial growth factor (VEGF) receptors on tumor cells that 
bind VEGF165 via its exon 7-encoded domain. J Biol Chem 1996;271:5761-7.

38. Schiller JH, Bittner G. Potentiation of platinum antitumor effects in human lung tumor xenografts by the angiogenesis inhibitor squalamine: effects on tumor neovascularization. Clin Cancer Res 1999;5:4287-94.

39. Mueller MD, Vigne JL, Minchenko A, Lebovic DI, Leitman DC, Taylor RN. Regulation of vascular endothelial growth factor (VEGF) gene transcription by estrogen receptors alpha and beta. Proc Natl Acad Sci USA 2000;97:10972-7.

40. Mauceri HJ, Hanna NN, Beckett MA, et al. Combined effects of angiostatin and ionizing radiation in antitumour therapy. Nature 1998; 394:287-91.

41. Williams JI, Mangold G, Zasloff M, Von Hoff DD. Evaluation of the natural aminosterol squalamine as adjunctive chemotherapy in the MX1 breast cancer xenograft model. Breast Cancer Res Treat 1997;46:723-33.

42. DeVita VT Jr, Hellman S, Rosenberg SA. Cancer: Principles \& Practice of Oncology. New York, NY: Lippincott-Raven, 2000.

43. DiSaia P, Bloss J. Treatment of ovarian cancer: new strategies. Gynecol Oncol 2003;90(2 Suppl):S24-32.

44. Ozols RF. Paclitaxel plus carboplatin in the treatment of ovarian cancer. Sem Oncol 1999;26:84-9.

45. Stiff, PJ. The challenge of treating advanced ovarian cancer. Cancer Mgmt 1997;2:1-5.

46. Lewis G, Figari I, Fendly B, et al. Differential responses of human tumor cell lines to anti-p185HER2 monoclonal antibodies. Cancer Immunol Immunother 1993;37:255-63.

47. Zebrowski BK, Liu W, Ramirez K, Akagi Y, Mills GB, Ellis LM. Markedly elevated levels of vascular endothelial growth factor in malignant ascites. Ann Surg Oncol 1999;6:373-8.
48. Kinney WA, Xhang X, Williams JI, et al. The challenge of treating advanced ovarian cancer. Organic Lett 2000;2:2921-2.

49. Bhargava P, Marshall JL, Dahut W, et al. A phase I and pharmacokinetic study of squalamine, a novel antiangiogenic agent, in patients with advanced cancers. Clin Cancer Res 2001;7:3912-9.

50. Hao D, Hammond LA, Eckhardt SG, et al. A phase I and pharmacokinetic study of squalamine, an aminosterol angiogenesis inhibitor. Clin Cancer Res 2003;9:2465-71.

51. Herbst RS, Hammond LA, Carbone DP, et al. A Phase I/IIA trial of continuous five-day infusion of squalamine lactate (MSI-1256F) plus carboplatin and paclitaxel in patients with advanced non-small cell lung cancer. Clin Cancer Res 2003;9:4108-15.

52. Davidson SA, Chap L, Pietras R, et al. A phase IIA trial of continuous 5-day infusions of MSI-1256F (squalamine lactate) plus carboplatin for therapy of persistant or recurrent advanced ovarian cancer. Proc Am Soc Clin Oncol 2002;9:2465-71.

53. D'Angelo G, Struman I, Martial J, Weiner R. Activation of mitogenactivated protein kinases by vascular endothelial growth factor and basic fibroblast growth factor in capillary endothelial cells is inhibited by the antiangiogenic factor 16-kd N-terminal fragment of prolactin. Proc Natl Acad Sci USA 1995;92:6374-8.

54. Pietras RJ, Arboleda J, Reese DM, et al. HER-2 tyrosine kinase pathway targets estrogen receptor and promotes hormone-independent growth in human breast cancer cells. Oncogene 1995;10:2435-46.

55. Pietras RJ, Poen JC, Gallardo D, Wongvipat PN, Lee HJ, Slamon DJ. Monoclonal antibody to HER-2/neureceptor modulates repair of radiationinduced DNA damage and enhances radiosensitivity of human breast cancer cells overexpressing this oncogene. Cancer Res 1999;59:1347-55.

Received September 13, 2004; accepted January 13, 2005 


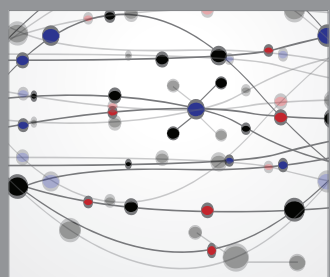

The Scientific World Journal
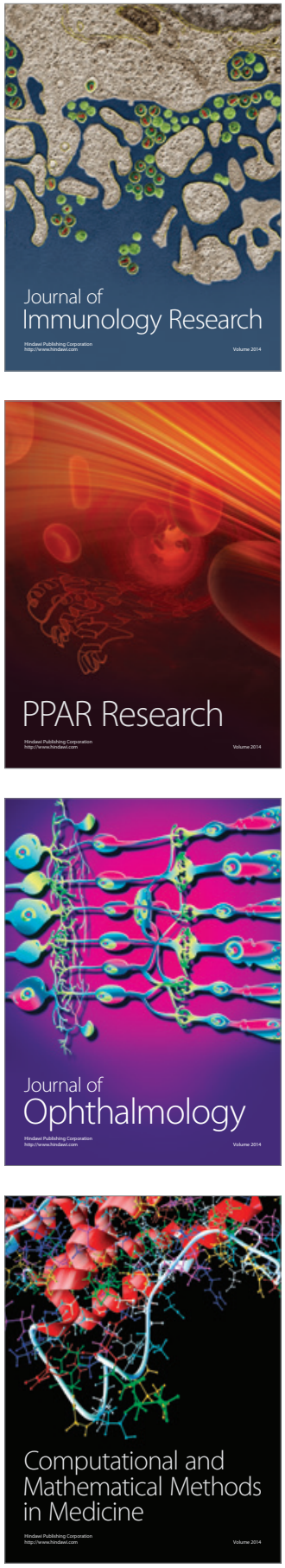

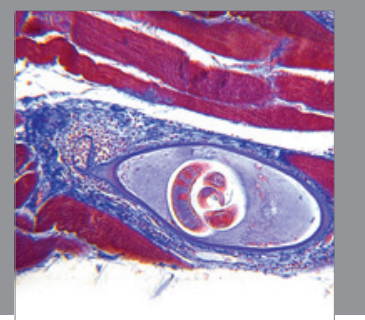

Gastroenterology

Research and Practice
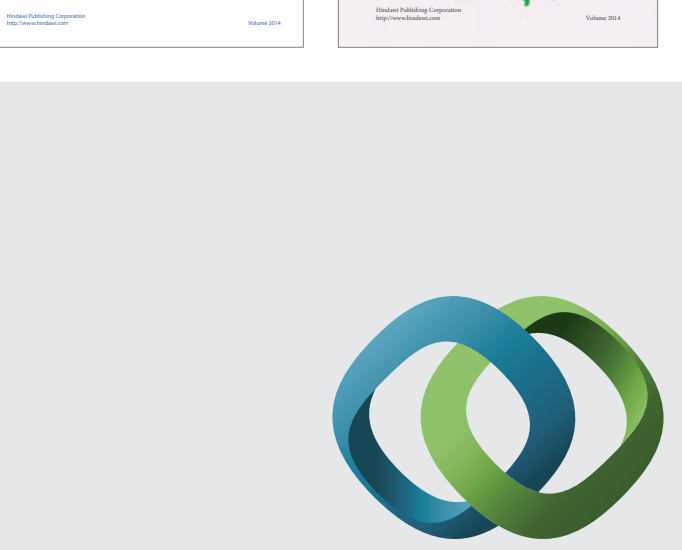

\section{Hindawi}

Submit your manuscripts at

http://www.hindawi.com
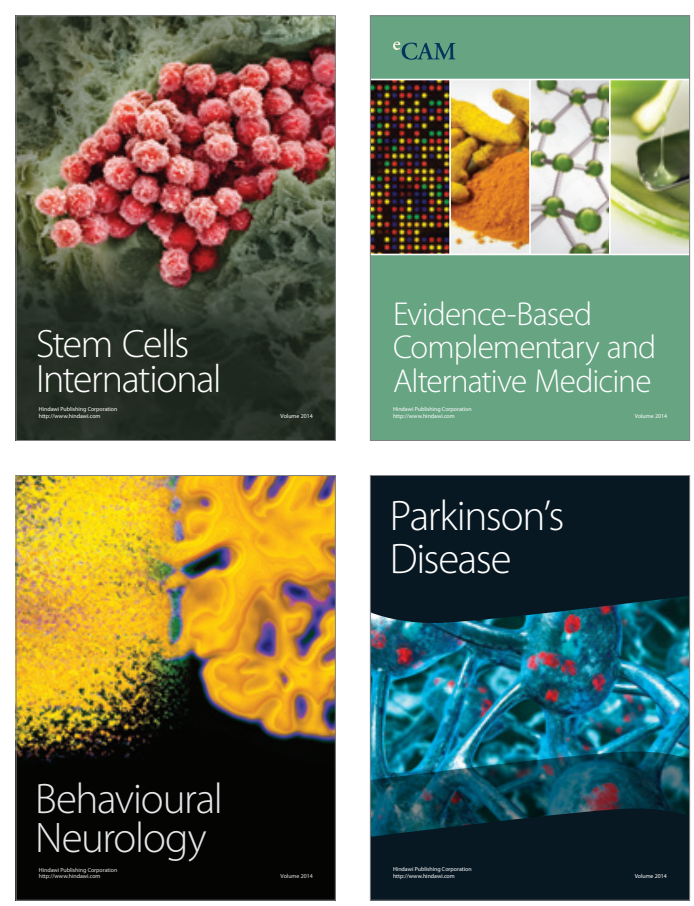

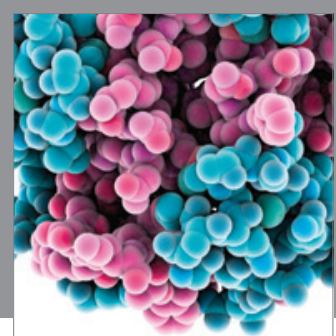

Journal of
Diabetes Research

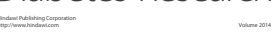

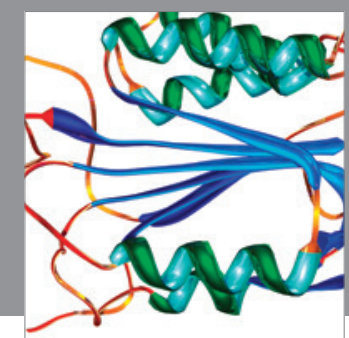

Disease Markers
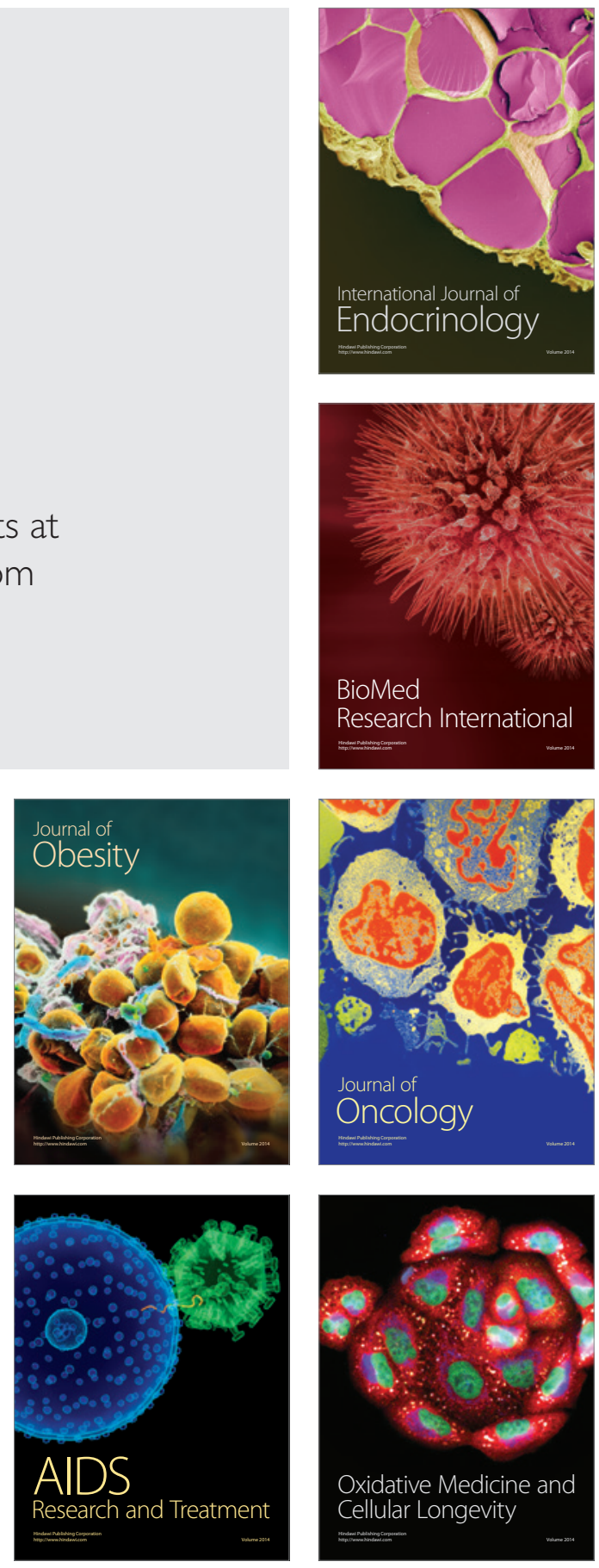